\title{
Inclination towards research and the pursuit of a research career among medical students: an international cohort study
}

Tam Cam Ha ${ }^{1,2^{*}}$, Sheryl $\mathrm{Ng}^{3}$, Cynthia Chen ${ }^{3}$, Sook Kwin Yong ${ }^{1}$, Gerald C. H. Koh ${ }^{3}$, Say Beng Tan ${ }^{1}$, Rahul Malhotra ${ }^{1}$, Fernando Altermatt ${ }^{4}$, Arnfinn Seim ${ }^{5}$, Aya Biderman ${ }^{6}$, Torres Woolley ${ }^{7}$ and Truls Østbye ${ }^{1,8}$

\begin{abstract}
Background: Involvement of clinicians in biomedical research is imperative for the future of healthcare. Several factors influence clinicians' inclination towards research: the medical school experience, exposure to research article reading and writing, and knowledge of research. This cohort study follows up medical students at time of graduation to explore changes in their inclination towards research and pursuing a research career compared to their inclination at time of entry into medical school.

Methods: Students from medical schools in six different countries were enrolled in their first year of school and followed-up upon graduation in their final year. Students answered the same self-administered questionnaire at both time points. Changes in inclination towards research and pursuing a research career were assessed. Factors correlated with these changes were analysed.

Results: Of the 777 medical students who responded to the study questionnaire at entry into medical school, 332 (42.7\%) completed the follow-up survey. Among these 332 students, there was no significant increase in inclination towards research or pursuing a research career over the course of their medical schooling. Students from a United States based school, in contrast to those from schools other countries, were more likely to report having research role models to guide them $(51.5 \%$ vs. $0 \%-26.4 \%)$ and to have published in a peer-reviewed journal (75.7\% vs. 8.9\%-45\%). Absence of a role model was significantly associated with a decrease in inclination towards research, while an increased desire to learn more about statistics was significantly associated with an increase in inclination towards pursuing a research career.

Conclusion: Most medical students did not experience changes in their inclination towards research or pursuing a research career over the course of their medical schooling. Factors that increased their inclination to undertaking research or pursuing a research career were availability of a good role model, and a good knowledge of both the research process and the analytical tools required.
\end{abstract}

Keywords: Inclination, Medical students, Research career, Research, Cohort

\footnotetext{
* Correspondence: tamha@uow.edu.au

'Duke-NUS Medical School, National University of Singapore, Singapore,

Singapore

${ }^{2}$ The University of Wollongong, Wollongong, Australia

Full list of author information is available at the end of the article
}

(c) The Author(s). 2018 Open Access This article is distributed under the terms of the Creative Commons Attribution 4.0 International License (http://creativecommons.org/licenses/by/4.0/), which permits unrestricted use, distribution, and reproduction in any medium, provided you give appropriate credit to the original author(s) and the source, provide a link to the Creative Commons license, and indicate if changes were made. The Creative Commons Public Domain Dedication waiver (http://creativecommons.org/publicdomain/zero/1.0/) applies to the data made available in this article, unless otherwise stated. 


\section{Background}

Biomedical research has the potential to generate positive health and economic outcomes [1]. However, studies across many countries have reported a decreasing trend of physicians undertaking clinical research [2]. Since 2000, the number of physician-scientists has not kept pace with the overall growth of the medical research community [3]. The progress of biomedical research is highly dependent on physicians being actively engaged in research. Physicians are more able to pursue clinically relevant research as they are in regular contact with patients, have a deeper understanding and appreciation of diseases and the clinical environment.

In view of the decreasing number of physicianscientists, various research councils and institutes across different countries are increasing their focus on training 'clinician scientists' (medical graduates who primarily pursue a career in biomedical research) and 'clinician investigators' (medical graduates who primarily are involved in patient care, but are also involved in research) [4].

Individual student experiences in medical school are the most important determinant in clinicians choosing to pursue a career in research and cultivating a research interest [5-7]. For example, several key factors associated with a career in research include participation in research projects or electives and authoring research papers while in medical school [8], involvement in research training and experience while in medical school [9], and the desire to pursue a graduate research degree along with a medical degree [10]. However, a recent study showed only a minority of first-year medical students across different countries and different medical schools were inclined towards a career in research [4].

This article reports on medical students' inclination towards a research career at the time of graduation, involving the same cohort as Tan et al. [4]. In that original study, medical students were assessed in terms of their confidence with biomedical research methodology, ability to interpret and understand biomedical literature, and their inclination towards research at the point of entry into medical school. The objective of this followup study is to determine whether medical students from different medical schools worldwide experienced any changes in their inclination towards research and the pursuit of a research career from first-year to the time of graduation, and which factors may have encouraged or discouraged such changes in inclinations.

\section{Methods}

This prospective cohort study is the follow-up to a previously published baseline survey [4]. Medical schools from eleven universities in ten countries across five continents collaborated on this comparison study. The study design, methodology and study implementation have been previously documented [4]. In brief, a selfadministered, non- anonymised questionnaire was used for a baseline study of first year medical students. The identical questionnaire was used for the prospective follow-up in students' final year. The inclination of students towards research and a biomedical research career were assessed by comparing the questions at the two time points (first year medical school vs final year medical school). The pilot-tested questionnaire was developed by the study team after extensive, iterative discussions in person, via telephone and email, and then later translated from English into three languages (Portuguese, Spanish and Hebrew). Questions included exposure to medical research, experience in performing medical research, and perceived research capabilities.

\section{Follow-up}

The medical students who responded to the baseline questionnaires were re-contacted near time of graduation. The questionnaire was identical as used in the baseline survey but with minor modifications, as each site required minor variations in procedures for administering the questionnaires due to differences in duration and course structure for each medical school. The minor modifications of the questionnaire were paraphrasing the questions in the baseline questionnaire with suitable tense used so as to be more grammatically appropriate to the final year medical students. The site investigators administered the questionnaire to final-year, graduating students during one of their compulsory classes, in person. The students then completed the questionnaires individually. The hard copies of the completed questionnaires were sent to the study team in Singapore for data analysis. Study participation was voluntary and informed written consent obtained from each student. Ethics approval was obtained from the NUS Institutional Review Board (NUS-IRB Ref No 08-274). Each site also obtained the necessary Institutional Review Board/Ethics Committee approval as required by the institution and country's regulations.

\section{Statistical analysis}

Descriptive summary statistics were computed for each university, and the data combined for all universities. Demographic data were taken from the baseline study while the curriculum data were taken from the followup survey. Chi-square tests were used to test bivariate associations between institutions and respondent characteristics. The categories relating to understanding and capabilities in research were biostatistics, research ethics and study design. Changes in inclination towards research and biomedical career were defined as changes in inclination from beginning to end of medical school 
(first and final year). Respondents who went from a 'No' to 'Yes'/ 'Undecided' and 'Undecided' to 'Yes' were deemed to have experienced an increase in inclination, while respondents who went from a 'Yes' to 'No'/ 'Undecided' and 'Undecided' to 'No' were deemed to have experienced a decrease in inclination.

Multinomial logistic regression was used to identify factors associated with two outcomes of interest; increase or decrease in inclination towards research, and increase or decrease inclination towards a research career (with no change in inclination as the reference comparison). A stepwise approach was used to remove any predictors which were not significant and odds ratios (OR) with confidence intervals reported. A sensitivity analysis was carried out for robustness of results: missing data of non-respondents in the follow-up survey was entered with their baseline information and full analyses were again performed. All statistical analyses were performed using R software (R. RStudio, Inc., Boston, MA, USA 2015). Significance was set at $p<0.05$ for all analyses.

\section{Results}

Among the 11 universities in 10 countries that were part of the original study, 7 universities from 6 countries responded to the follow-up. These universities were: James Cook University (JCU) Australia; Pontificia Universidad Catolica de Chile (PUC) Chile; Ben-Gurion University (BGU) Israel; Duke University USA; Norwegian University of Science and Technology (NTNU) Norway; and the National University of Singapore (NUS) (students from both Yong Loo Lin School of Medicine and Duke-NUS Medical School) Singapore (Table 1). Singapore data were presented as a combination of the two schools because the sample size for Duke-Nus Medical School was too small to be presented as a stand-alone site.

A total of 777 responses were provided in the baseline survey; of these, $332(42.7 \%)$ responded to the final year survey. The demographics of the students who responded to the follow-up study are described in Table 1 . The median age at baseline was 19 years (range; 16-33 years), with BGU and Duke University having the highest median age of 24 years, and JCU Australia the lowest at 17 years. Duke University (USA) and DukeNUS (Singapore) were the only institutions with solely graduate students, so most students $(73.1 \%)$ overall did not have a previous degree. There was no significant difference in baseline characteristics between the respondents and non-respondents of this follow-up survey, but most of the non-respondents did not have any prior experience with research (data not shown).

Most students experienced no change in inclination towards research except JCU (44.4\% vs. $37.8 \%)$ and PUC
(40.0\% vs. $35.0 \%)$, where more students increased their inclination towards research compared to those who had no change in inclination towards research. Also, most students reported no change in their inclination towards research careers.

The majority of students had performed some research during the course of their medical school but had not taken any additional courses in research or ethics outside their regular curriculum; this was observed across all the institutions. While few students overall had published an article in peer-reviewed journals when in medical school, Duke University students reported otherwise with $75.7 \%$ having done so.

Most students indicated that they did not have any role model to guide their research during the course of the medical programme except Duke University, where more than half $(51.4 \%$ vs. $0 \%-26.4 \%)$ of the students reported in the affirmative. In addition, Duke University students envisioned having the highest percentage of their postgraduate work time spent undertaking research (mean: $23 \%$ vs. $7.7 \%-11.6 \%$ ).

Table 2 presents the change in the self-reported capabilities and confidence of the medical students. While no significant change in students' understanding or confidence towards research methods was observed, BGU had the highest proportion of increased understanding in biostatistics, capabilities in study design and data analysis, while NTNU and NUS had high proportions of students with increased understanding of research ethics, and Duke University recorded the highest proportion of students with increased confidence in publication ethics. The aspects which various medical schools' students showed consistent trend in increasing confidence were understanding ethics terms found in research journals and evaluating diagnostic tests.

The only factor which was significantly associated with an increased inclination towards research was increased desire to learn biostatistics (Odds Ratio (OR) 1.68; Confidence Interval (CI) 1.03-2.74) (Table 3). However, after adjusting for gender, age and institution, increased desire to learn biostatistics was no longer statistically significant. Meanwhile, absence of a role model and lack of understanding of study design terms described in journal articles were significantly associated with a decrease in inclination towards research (Table 3). After multivariate adjustment, only absence of a research role model remained statistically significant (OR 0.37; CI 0. 18-0.79).

Table 4 summarises the capability and confidence factors that were significantly associated with a change in inclination towards a research career. Students who reported an increased desire to learn more about biostatistics and an increased knowledge of manipulation of statistics were more likely to experience an increased 
Table 1 Demographic profiles, research inclination and previous research experience of the medical students from various medical schools

\begin{tabular}{|c|c|c|c|c|c|c|c|}
\hline & \multirow[b]{2}{*}{ Total } & \multicolumn{6}{|l|}{ Institutions $^{a}$} \\
\hline & & $\mathrm{JCU}$ & PUC & $B G U$ & DUMC & NTNU & NUS \\
\hline First year & 777 & 167 & 98 & 41 & 82 & 108 & 281 \\
\hline Final year & 332 & 45 & 20 & 29 & 37 & 47 & 154 \\
\hline \multicolumn{8}{|l|}{ N (\%) } \\
\hline \multicolumn{8}{|l|}{ Age at first year } \\
\hline Median & 19 & 17 & 18 & 24 & 24 & 22 & 19 \\
\hline (Min, Max) & $(16,33)$ & $(16,22)$ & $(17,23)$ & $(19,27)$ & $(22,33)$ & $(19,25)$ & $(18,28)$ \\
\hline \multicolumn{8}{|l|}{ Gender } \\
\hline Female & $179(53.9)$ & $25(55.6)$ & $9(45.0)$ & $12(41.4)$ & $15(40.5)$ & $35(74.5)$ & $83(53.9)$ \\
\hline Male & $153(46.1)$ & $20(44.4)$ & $11(55.0)$ & $17(58.6)$ & $22(59.5)$ & $12(25.5)$ & $71(46.1)$ \\
\hline \multicolumn{8}{|c|}{ Highest Academic Qualification } \\
\hline No previous degree & 239/327 (73.1) & 40/44 (90.9) & $19(95.0)$ & $25 / 28(89.3)$ & $0(0.0)$ & $35 / 45(77.8)$ & $120 / 153(78.4)$ \\
\hline Diploma & $18 / 327(5.5)$ & $3 / 44(6.8)$ & $1(5.0)$ & $1 / 28(3.6)$ & $0(0.0)$ & $3 / 45(6.7)$ & $11 / 153(7.2)$ \\
\hline Bachelors & $61 / 327(18.7)$ & $0(0.0)$ & $0(0.0)$ & $2 / 28(7.1)$ & $32(86.5)$ & $7 / 45(15.6)$ & 19/153 (12.4) \\
\hline Masters & $7 / 327(2.1)$ & $1 / 44(2.3)$ & $0(0.0)$ & $0(0.0)$ & $4(10.8)$ & $0(0.0)$ & 2/153 (1.3) \\
\hline PhD \& above & 2/327 (0.6) & $0(0.0)$ & $0(0.0)$ & $0(0.0)$ & $1(2.7)$ & $0(0.0)$ & $1 / 153(0.7)$ \\
\hline \multicolumn{8}{|c|}{ Inclination towards research ${ }^{b}$} \\
\hline No change & 133/323 (41.2) & $17(37.8)$ & $7(35.0)$ & $11 / 28(39.3)$ & 17/35 (48.6) & $25(53.2)$ & $56 / 148(37.8)$ \\
\hline Decrease & 90/323 (27.9) & $8(17.8)$ & $5(25.0)$ & $7 / 28(25.0)$ & $6 / 35(17.1)$ & $14(29.8)$ & $50 / 148(33.8)$ \\
\hline Increase & 100/323 (31.0) & $20(44.4)$ & $8(40.0)$ & $10 / 28(35.7)$ & $12 / 35(34.3)$ & $8(17.0)$ & $42 / 148(28.4)$ \\
\hline \multicolumn{8}{|c|}{ Inclination towards a biomedical career ${ }^{\mathrm{b}}$} \\
\hline No change & 137/329 (41.6) & 17/44 (38.6) & $3(15.0)$ & $11 / 28(39.3)$ & 14/36 (38.9) & $25(53.2)$ & $67(43.5)$ \\
\hline Decrease & 118/329 (35.9) & 18/44 (40.9) & $15(75.0)$ & $6 / 28(21.4)$ & $8 / 36(22.2)$ & $10(21.3)$ & 61 (39.6) \\
\hline Increase & $74 / 329(22.5)$ & $9 / 44(20.5)$ & $2(10.0)$ & $11 / 28(39.3)$ & 14/36 (38.9) & $12(25.5)$ & $26(16.9)$ \\
\hline \multicolumn{8}{|c|}{ Done research during medical school } \\
\hline No & $41 / 326(12.6)$ & $22(48.9)$ & $2(10.0)$ & $1(3.4)$ & $0(0.0)$ & $0(0.0)$ & 17/152 (11.2) \\
\hline Yes & 285/326 (87.4) & $23(51.1)$ & $18(90.0)$ & $28(96.6)$ & $37(100.0)$ & $47(100.0)$ & 135/152 (88.8) \\
\hline \multicolumn{8}{|c|}{ Taken a course in research during medical school outside regular curriculum } \\
\hline No & $255(76.8)$ & $41(91.1)$ & $18(90.0)$ & $17(58.6)$ & $20(54.1)$ & $41(87.2)$ & $118(76.6)$ \\
\hline Yes & $77(23.2)$ & $4(8.9)$ & $2(10.0)$ & $12(41.4)$ & $17(45.9)$ & $6(12.8)$ & $36(23.4)$ \\
\hline \multicolumn{8}{|c|}{ Taken a course in research ethics during medical school outside regular curriculum } \\
\hline No & 295/326 (90.2) & $42 / 44(95.4)$ & $20(100.0)$ & $23 / 28(82.1)$ & $25(67.6)$ & $47(100.0)$ & 138/151 (91.4) \\
\hline Yes & $32 / 326(9.8)$ & $2 / 44(4.6)$ & $0(0.0)$ & $5 / 28(17.9)$ & $12(32.4)$ & $0(0.0)$ & 13/151 (8.6) \\
\hline \multicolumn{8}{|c|}{ Had articles accepted or published in peer-reviewed journals while in medical school } \\
\hline No & $218(65.7)$ & $41(91.1)$ & $11(55.0)$ & $25(86.2)$ & $9(24.3)$ & $36(76.6)$ & $96(62.3)$ \\
\hline Yes & $114(34.3)$ & $4(8.9)$ & $9(45.0)$ & $4(13.8)$ & $28(75.7)$ & $11(23.4)$ & $58(37.7)$ \\
\hline \multicolumn{8}{|c|}{ Had an experience during medical school that put off respondent from research } \\
\hline No & 248/326 (74.9) & $36(80.0)$ & $6(30.0)$ & $28(96.6)$ & $22(59.5)$ & $42 / 46(91.3)$ & $114(74.0)$ \\
\hline Yes & $83 / 326(25.1)$ & $9(20.0)$ & $14(70.0)$ & $1(3.4)$ & $15(40.5)$ & $4 / 46(8.7)$ & $40(26.0)$ \\
\hline \multicolumn{8}{|c|}{ Had an experience during medical school that ignited interest in research } \\
\hline No & $230 / 326(70.3)$ & $26 / 43(60.5)$ & $9(45.0)$ & $22(75.9)$ & $18(48.6)$ & $35(74.5)$ & 120/151 (79.5) \\
\hline Yes & $97 / 326(29.7)$ & $17 / 43(39.5)$ & $11(55.0)$ & $7(24.1)$ & $19(51.4)$ & $12(25.5)$ & $31 / 151(20.5)$ \\
\hline
\end{tabular}


Table 1 Demographic profiles, research inclination and previous research experience of the medical students from various medical schools (Continued)

\begin{tabular}{|c|c|c|c|c|c|c|c|}
\hline & \multirow[b]{2}{*}{ Total } & \multicolumn{6}{|l|}{ Institutions $^{a}$} \\
\hline & & $\mathrm{JCU}$ & PUC & BGU & DUMC & NTNU & NUS \\
\hline \multicolumn{8}{|c|}{ Read biomedical journals at least once every 6 months } \\
\hline No & $163(49.1)$ & $15(33.3)$ & $10(50.0)$ & $14(48.3)$ & $13(35.1)$ & $17(36.2)$ & $94(61.0)$ \\
\hline Yes & $169(50.9)$ & $30(66.7)$ & $10(50.0)$ & $15(51.7)$ & $24(64.9)$ & $30(63.8)$ & $60(39.0)$ \\
\hline \multicolumn{8}{|c|}{ Presence of role model during medical school } \\
\hline No & 232/326 (73.7) & $31 / 40(77.5)$ & 15/15 (100.0) & $21 / 28(75.0)$ & $18(48.6)$ & $38(80.9)$ & 109/148 (73.6) \\
\hline Yes & $83 / 326(26.3)$ & $9 / 40(22.5)$ & $0 / 15(0.0)$ & $7 / 28(25.0)$ & $19(51.4)$ & $9(19.1)$ & $39 / 148(26.4)$ \\
\hline \multicolumn{8}{|c|}{ Percentage of work time envisioned to spend on research } \\
\hline Mean (SD) & $11.6(12.7)$ & $9.1(11.9)$ & $7.7(4.2)$ & $10.7(4.32)$ & $23.0(22.0)$ & $11.3(13.2)$ & $10.2(9.8)$ \\
\hline
\end{tabular}

a JCU James Cook University, PUC, Pontificia Universidad Catolica de Chile, BGU Ben-Gurion University, DUMC Duke University Medical Centre, NTNU Norwegian University of Science and Technology, NUS National University of Singapore (inclusive of Yong Loo Lin School of Medicine and from Duke-NUS Graduate Medical School)

bIncrease: No to Undecided or Yes, or Undecided to Yes; Decrease: Yes to Undecided or No; or Undecided to No

inclination towards a research career. After multivariate adjustment, only an increased desire to learn more about biostatistics remained significant (OR 2.58; 1.38-4.84). Meanwhile, the factors associated with decreased inclination towards research career were an understanding of data analysis, research ethics, knowledge of study design and potential biases (Table 4). In multivariate analysis, the ability to analyse data was significantly associated with a decrease in inclination towards research career (OR 0.49; 0.31-0.78).

\section{Discussion}

The initial investigation of inclination of medical students at entry into medical school from different parts of the world showed $35 \%$ had an inclination towards research and 25\% towards a research career [4]. At followup, overall, there was a slight decline in inclination; 31\% of students were inclined towards research and 23\% inclined towards a research career. The change in inclination towards research may possibly be due to the school's curricula, and the format of teaching in each of the medical schools.

Longitudinal studies such as the present study will assist in the assessment of current medical programmes for supporting students to undertake and be better motivated towards research, as well as improvements that could be made to curricula. It is alarming to know that year by year the numbers of dedicated academic posts are declining [6]. Thus, following our students as they complete their medical school and enter into residency allows a better understanding of their interests and the impact of the medical school curricula on research training.

While our findings suggest that medical students did not have any statistically significant changes in their interest towards research and pursuing a research career, there were a few schools with a larger proportion of students who increased their inclination towards various aspects of research. BGU in Israel provided the best learning experience to increase students' interest and confidence in research, while Duke University in USA and NUS in Singapore both improved students' confidence in publication ethics.

The study also showed that presence of a good research role model and knowledge in biostatistics and study design/research methodologies were key influences in changes of inclination towards research and research careers. It is known that when the ease of access to readily available information is present, the likelihood of students wanting to pursue a research opportunity also increases [5]. Good and reliable resources that are readily available are important in encouraging medical students to incorporate research into their activities.

There have been few other similar studies on factors that influence the pursuit of research or research careers preferences by medical students [11-13] and studies on numbers of medical students that have research career plans [14, 15]. Our study suggests a worrying trend of consistently low interest in research among medical students. Similarly, [3] Guelich et al. showed a declining trend of research intentions over the span of a decade between 1987 and 1997. Our follow-up study identifies some of the factors and barriers associated with interest in research, and these should be considered in reviewing medical curricula to allow for greater emphasis on the training of clinician scientists. In addition, a previous study suggested medical schools should improve their school curricula by portraying medical research as a rewarding and enjoyable career [3], while a more recent study suggested the curriculum could incorporate compulsory group-based research activities that are 
Table 2 Proportion of medical students who changed in agreement to different statements and confidence on various aspects biomedical research, $\mathrm{n}(\%)$




Table 2 Proportion of medical students who changed in agreement to different statements and confidence on various aspects biomedical research, $\mathrm{n}$ (\%) (Continued)

\begin{tabular}{|c|c|c|c|c|c|c|c|c|c|}
\hline & \multirow{3}{*}{$\begin{array}{l}\text { Total } \\
(n=332)\end{array}$} & \multicolumn{6}{|c|}{ Institutions $^{a}$} & \\
\hline & & & $\mathrm{JCU}$ & PUC & $B G U$ & DUMC & NTNU & NUS & \\
\hline & & & $(n=45)$ & $(n=20)$ & $(n=29)$ & $(n=37)$ & $(n=47)$ & $(n=154)$ & \\
\hline & Increased & $\begin{array}{l}141 / 328 \\
(43.0)\end{array}$ & $\begin{array}{l}26 \\
(57.8)\end{array}$ & $\begin{array}{l}11 \\
(55.0)\end{array}$ & $\begin{array}{l}16 / 28 \\
(57.1)\end{array}$ & $\begin{array}{l}12 \\
(32.4)\end{array}$ & $\begin{array}{l}25 / 46 \\
(54.3)\end{array}$ & $\begin{array}{l}51 / 152 \\
(33.6)\end{array}$ & \\
\hline \multirow[t]{2}{*}{$\begin{array}{l}\text { Interpreting the implications to clinical practice } \\
\text { for a given result from a statistical analysis }\end{array}$} & Decreased & $\begin{array}{l}24 / 330 \\
(7.3)\end{array}$ & $\begin{array}{l}1 \\
(2.2)\end{array}$ & $\begin{array}{l}2 \\
(10.0)\end{array}$ & $\begin{array}{l}1 / 28 \\
(3.6)\end{array}$ & $1(2.7)$ & $\begin{array}{l}2 \\
(4.3)\end{array}$ & $\begin{array}{l}17 / 153 \\
(11.1)\end{array}$ & 0.097 \\
\hline & Increased & $\begin{array}{l}133 / 330 \\
(40.3)\end{array}$ & $\begin{array}{l}19 \\
(42.2)\end{array}$ & $\begin{array}{l}12 \\
(60.0)\end{array}$ & $\begin{array}{l}15 / 28 \\
(53.6)\end{array}$ & $\begin{array}{l}15 \\
(40.5)\end{array}$ & $\begin{array}{l}22 \\
(46.8)\end{array}$ & $\begin{array}{l}50 / 153 \\
(32.7)\end{array}$ & \\
\hline \multirow[t]{2}{*}{$\begin{array}{l}\text { Assessing if the correct statistical procedure } \\
\text { was used to answer a research question }\end{array}$} & Decreased & $\begin{array}{l}45 / 327 \\
(13.8)\end{array}$ & $\begin{array}{l}7 / 44 \\
(15.9)\end{array}$ & $\begin{array}{l}9 / 19 \\
(47.4)\end{array}$ & $\begin{array}{l}3 / 28 \\
(10.7)\end{array}$ & $3(8.1)$ & $\begin{array}{l}5 / 46 \\
(10.9)\end{array}$ & $\begin{array}{l}18 / 153 \\
(11.8)\end{array}$ & $<0.001^{*}$ \\
\hline & Increased & $\begin{array}{l}94 / 327 \\
(28.7)\end{array}$ & $\begin{array}{l}7 / 44 \\
(15.9)\end{array}$ & $\begin{array}{l}4 / 19 \\
(21.1)\end{array}$ & $\begin{array}{l}12 / 28 \\
(42.9)\end{array}$ & $\begin{array}{l}12 \\
(32.4)\end{array}$ & $\begin{array}{l}10 / 46 \\
(21.7)\end{array}$ & $\begin{array}{l}49 / 153 \\
(32.0)\end{array}$ & \\
\hline \multirow[t]{2}{*}{$\begin{array}{l}\text { Identifying the factors that influence the } \\
\text { adequacy of a study's sample size }\end{array}$} & Decreased & $\begin{array}{l}65 / 330 \\
(19.7)\end{array}$ & $\begin{array}{l}11 \\
(24.4)\end{array}$ & $\begin{array}{l}13 \\
(65.0)\end{array}$ & $\begin{array}{l}4 / 28 \\
(14.3)\end{array}$ & $2(5.4)$ & $\begin{array}{l}6 \\
(12.8)\end{array}$ & $\begin{array}{l}29 / 153 \\
(19.0)\end{array}$ & $<0.001^{*}$ \\
\hline & Increased & $\begin{array}{l}84 / 330 \\
(25.5)\end{array}$ & $\begin{array}{l}9 \\
(20.0)\end{array}$ & $\begin{array}{l}1 \\
(5.0)\end{array}$ & $\begin{array}{l}6 / 28 \\
(21.4)\end{array}$ & $\begin{array}{l}13 \\
(35.1)\end{array}$ & $\begin{array}{l}19 \\
(40.4)\end{array}$ & $\begin{array}{l}36 / 153 \\
(23.5)\end{array}$ & \\
\hline \multirow[t]{2}{*}{ Evaluating diagnostic tests } & Decreased & $\begin{array}{l}24 / 327 \\
(7.3)\end{array}$ & $\begin{array}{l}2 / 43 \\
(4.7)\end{array}$ & $\begin{array}{l}2 \\
(10.0)\end{array}$ & $\begin{array}{l}2 \\
(6.9)\end{array}$ & $0(0.0)$ & $\begin{array}{l}4 \\
(8.5)\end{array}$ & $\begin{array}{l}14 / 151 \\
(9.3)\end{array}$ & 0.154 \\
\hline & Increased & $\begin{array}{l}152 / 327 \\
(46.5)\end{array}$ & $\begin{array}{l}14 / 43 \\
(32.6)\end{array}$ & $\begin{array}{l}10 \\
(50.0)\end{array}$ & $\begin{array}{l}18 \\
(62.1)\end{array}$ & $\begin{array}{l}21 \\
(56.8)\end{array}$ & $\begin{array}{l}25 \\
(53.2)\end{array}$ & $\begin{array}{l}64 / 151 \\
(42.4)\end{array}$ & \\
\hline \multirow[t]{2}{*}{$\begin{array}{l}\text { Analyzing the data to find association between } \\
\text { two variables }\end{array}$} & Decreased & $\begin{array}{l}52 / 328 \\
(15.9)\end{array}$ & $\begin{array}{l}8 / 44 \\
(18.2)\end{array}$ & $\begin{array}{l}5 \\
(25.0)\end{array}$ & $\begin{array}{l}3 \\
(10.3)\end{array}$ & $1(2.7)$ & $\begin{array}{l}6 \\
(12.8)\end{array}$ & $\begin{array}{l}29 / 151 \\
(19.2)\end{array}$ & $0.039^{*}$ \\
\hline & Increased & $\begin{array}{l}88 / 328 \\
(26.8)\end{array}$ & $\begin{array}{l}4 / 44 \\
(9.1)\end{array}$ & $\begin{array}{l}6 \\
(30.0)\end{array}$ & $\begin{array}{l}12 \\
(41.4)\end{array}$ & $\begin{array}{l}13 \\
(35.1)\end{array}$ & $\begin{array}{l}12 \\
(25.5)\end{array}$ & $\begin{array}{l}41 / 151 \\
(27.2)\end{array}$ & \\
\hline \multirow[t]{2}{*}{$\begin{array}{l}\text { Analyzing the data to find correlation between } \\
\text { two variables }\end{array}$} & Decreased & $\begin{array}{l}57 / 328 \\
(17.4)\end{array}$ & $\begin{array}{l}7 / 44 \\
(15.9)\end{array}$ & $\begin{array}{l}6 \\
(30.0)\end{array}$ & $\begin{array}{l}2 \\
(6.9)\end{array}$ & $1(2.7)$ & $\begin{array}{l}9 \\
(19.1)\end{array}$ & $\begin{array}{l}32 / 151 \\
(21.2)\end{array}$ & $<0.001^{*}$ \\
\hline & Increased & $\begin{array}{l}84 / 328 \\
(25.6)\end{array}$ & $\begin{array}{l}4 / 44 \\
(9.1)\end{array}$ & $\begin{array}{l}5 \\
(25.0)\end{array}$ & $\begin{array}{l}15 \\
(51.7)\end{array}$ & $\begin{array}{l}12 \\
(32.4)\end{array}$ & $\begin{array}{l}9 \\
(19.1)\end{array}$ & $\begin{array}{l}39 / 151 \\
(25.8)\end{array}$ & \\
\hline \multicolumn{10}{|l|}{ Research Ethics } \\
\hline \multirow[t]{2}{*}{$\begin{array}{l}\text { Applying ethical principles (e.g. confidentiality, } \\
\text { beneficence, non-maleficence, autonomy) }\end{array}$} & Decreased & $\begin{array}{l}23 / 323 \\
(7.1)\end{array}$ & $\begin{array}{l}3 \\
(6.7)\end{array}$ & $\begin{array}{l}1 / 19 \\
(5.3)\end{array}$ & $\begin{array}{l}3 / 27 \\
(11.1)\end{array}$ & $1(2.7)$ & $\begin{array}{l}6 \\
(12.8)\end{array}$ & $\begin{array}{l}9 / 148 \\
(6.1)\end{array}$ & $0.021^{*}$ \\
\hline & Increased & $\begin{array}{l}64 / 323 \\
(19.8)\end{array}$ & $\begin{array}{l}6 \\
(13.3)\end{array}$ & $\begin{array}{l}3 / 19 \\
(15.8)\end{array}$ & $\begin{array}{l}3 / 27 \\
(11.1)\end{array}$ & $\begin{array}{l}14 \\
(37.8)\end{array}$ & $\begin{array}{l}15 \\
(31.9)\end{array}$ & $\begin{array}{l}23 / 148 \\
(15.5)\end{array}$ & \\
\hline \multicolumn{10}{|l|}{ Research Ethics } \\
\hline \multirow[t]{2}{*}{$\begin{array}{l}\text { Applying for an approval from an Institutional } \\
\text { Review Board (IRB) / Ethics Committee }\end{array}$} & Decreased & $\begin{array}{l}21 / 324 \\
(6.5)\end{array}$ & $\begin{array}{l}7 \\
(15.6)\end{array}$ & $\begin{array}{l}3 \\
(15.0)\end{array}$ & $\begin{array}{l}1 / 26 \\
(3.8)\end{array}$ & $1(2.7)$ & $\begin{array}{l}2 \\
(4.3)\end{array}$ & $\begin{array}{l}7 / 149 \\
(4.7)\end{array}$ & $0.043^{*}$ \\
\hline & Increased & $\begin{array}{l}146 / 324 \\
(45.1)\end{array}$ & $\begin{array}{l}14 \\
(31.1)\end{array}$ & $\begin{array}{l}5 \\
(25.0)\end{array}$ & $\begin{array}{l}10 / 26 \\
(38.5)\end{array}$ & $\begin{array}{l}21 \\
(56.8)\end{array}$ & $\begin{array}{l}26 \\
(55.3)\end{array}$ & $\begin{array}{l}70 / 149 \\
(47.0)\end{array}$ & \\
\hline \multirow[t]{2}{*}{$\begin{array}{l}\text { Criteria to justify authorship in research journal } \\
\text { publications }\end{array}$} & Decreased & $\begin{array}{l}27 / 324 \\
(8.3)\end{array}$ & $\begin{array}{l}7 \\
(15.6)\end{array}$ & $\begin{array}{l}1 / 19 \\
(5.3)\end{array}$ & $\begin{array}{l}3 / 28 \\
(10.7)\end{array}$ & $1(2.7)$ & $\begin{array}{l}0 \\
(0.0)\end{array}$ & $\begin{array}{l}15 / 149 \\
(10.1)\end{array}$ & $0.005^{*}$ \\
\hline & Increased & $\begin{array}{l}113 / 324 \\
(34.9)\end{array}$ & $\begin{array}{l}5 \\
(11.1)\end{array}$ & $\begin{array}{l}5 / 19 \\
(26.3)\end{array}$ & $\begin{array}{l}8 / 28 \\
(28.6)\end{array}$ & $\begin{array}{l}19 \\
(51.4)\end{array}$ & $\begin{array}{l}20 / 46 \\
(43.5)\end{array}$ & $\begin{array}{l}56 / 149 \\
(37.6)\end{array}$ & \\
\hline \multirow[t]{2}{*}{$\begin{array}{l}\text { Awareness of the actions that constitute } \\
\text { publication misconduct }\end{array}$} & Decreased & $\begin{array}{l}47 / 319 \\
(14.7)\end{array}$ & $\begin{array}{l}5 \\
(11.1)\end{array}$ & $\begin{array}{l}4 \\
(20.0)\end{array}$ & $\begin{array}{l}3 / 28 \\
(10.7)\end{array}$ & $2(5.4)$ & $\begin{array}{l}3 / 46 \\
(6.5)\end{array}$ & $\begin{array}{l}30 / 143 \\
(21.0)\end{array}$ & $0.017^{*}$ \\
\hline & Increased & $\begin{array}{l}94 / 319 \\
(29.5)\end{array}$ & $\begin{array}{l}9 \\
(20.0)\end{array}$ & $\begin{array}{l}3 \\
(15.0)\end{array}$ & $\begin{array}{l}6 / 28 \\
(21.4)\end{array}$ & $\begin{array}{l}18 \\
(48.6)\end{array}$ & $\begin{array}{l}16 / 46 \\
(34.8)\end{array}$ & $\begin{array}{l}42 / 143 \\
(29.4)\end{array}$ & \\
\hline \multicolumn{10}{|l|}{ Study Design } \\
\hline \multirow[t]{2}{*}{$\begin{array}{l}\text { Knowledge of the advantages and } \\
\text { disadvantages of each study design }\end{array}$} & Decreased & $\begin{array}{l}19 / 327 \\
(5.8)\end{array}$ & $\begin{array}{l}4 / 43 \\
(9.3)\end{array}$ & $\begin{array}{l}1 \\
(5.0)\end{array}$ & $\begin{array}{l}1 \\
(3.4)\end{array}$ & $0(0.0)$ & $\begin{array}{l}1 \\
(2.1)\end{array}$ & $\begin{array}{l}12 / 151 \\
(7.9)\end{array}$ & $0.081^{*}$ \\
\hline & Increased & $\begin{array}{l}145 / 327 \\
(44.3)\end{array}$ & $\begin{array}{l}12 / 43 \\
(27.9)\end{array}$ & $\begin{array}{l}14 \\
(70.0)\end{array}$ & $\begin{array}{l}16 \\
(55.2)\end{array}$ & $\begin{array}{l}17 \\
(45.9)\end{array}$ & $\begin{array}{l}22 \\
(46.8)\end{array}$ & $\begin{array}{l}64 / 151 \\
(42.4)\end{array}$ & \\
\hline
\end{tabular}


Table 2 Proportion of medical students who changed in agreement to different statements and confidence on various aspects biomedical research, $\mathrm{n}(\%)$ (Continued)



aJCU James Cook University, PUC Pontificia Universidad Catolica de Chile, BGU Ben-Gurion University, DUMC Duke University Medical Centre, NTNU Norwegian University of Science and Technology, NUS National University of Singapore (inclusive of Yong Loo Lin School of Medicine and from Duke-NUS Graduate Medical School)

${ }^{\mathrm{b}}$ Based on Chi-square test

'Remaining data not reported in the table had 'no change' in those statements

${ }^{*} p$-value $<0.05$ were statistically significant

well supported as a way to motivate students to undertake research and potentially careers in research [7].

In order to stem the declining interest of future clinicians towards research and research careers, current medical curricula should address barriers to student research, and adopt motivational strategies for research activity. Barriers such as inadequate funding for research, differential in pay between community and academic centres, and lack of proper initial mentorship during the transition period from resident to independent investigator have been noted as early as 2002 [16]. Barriers such as time and the perception that students would not receive appropriate acknowledgement for work contributed to a research project should also be addressed [13]. Early exposure to research may also help demystify the process of conducting research and render it more likely that research activities will be pursued during residency and potentially as a career [5].

\section{Limitations}

There are several limitations to this study. All capabilities and confidence towards research were selfreported. As expected, there could be bias in perceived knowledge of statistics and medical research among the medical students. Also, as this was a prospective cohort study, following students up from different medical schools across varying countries was challenging, with the loss to follow up being $57 \%$. Thus, the study is subjected to responder biases. Reasons for this significant loss were the long follow-up period, turnover of faculty staff, students being busy in their final year, and some students being posted to different training sites during their final year thus making it difficult to contact them for the survey. Other limitations include variations in medical school duration and follow-up across different countries. However, the follow-up process was standardised as much as possible by administering the questionnaire the same way it was at entry into medical school, and adjusting for institutional effects in multivariate models. Despite the limitations of missing data and a low follow up response rate, some cohorts demonstrated an increased inclination towards research of $22 \%$, with $35 \%$ were less inclined. These figures may be what can realistically be expected from a cohort of medical students in terms of their interest in pursuing research careers. Due to the varying numbers of students and the small number of students in some schools, this may limit the comparisons made among the schools. However, providing results by school as well as an aggregate is a starting point for further investigations as to why differences exists in the interests towards pursuing research degrees. Examination of differences in medical school curricula may allow more specific changes to be made to encourage and inspire students to pursue research careers. We also did not examined the effect of having a previous degree on their interest in pursuing biomedical research as there were only a small number of students $(26 \%)$ that had a previous degree before pursuing a medical degree. We

Table 3 Change in inclination towards research (multinomial logistic regression)

\begin{tabular}{|c|c|c|c|c|}
\hline \multirow[t]{2}{*}{ Factors } & \multicolumn{2}{|c|}{ Increase inclination towards research } & \multicolumn{2}{|c|}{ Decrease inclination towards research } \\
\hline & Bivariate (OR) & Multivariate $^{\mathrm{a}}(\mathrm{OR})$ & Bivariate (OR) & Multivariate $^{\mathrm{a}}(\mathrm{OR})$ \\
\hline Role model during medical school by final year & $0.93(0.52-1.67)$ & $0.86(0.46-1.61)$ & $0.40(0.20-0.80)^{*}$ & $0.37(0.18-0.79)^{*}$ \\
\hline Desire to learn more about biostatistics & $1.68(1.03-2.74)^{*}$ & $1.64(0.94-2.86)$ & $0.71(0.43-1.18)$ & $0.76(0.44-1.32)$ \\
\hline Understanding of study design terms in journal articles & $0.76(0.48-1.20)$ & - & $0.56(0.35-0.89)^{*}$ & \\
\hline
\end{tabular}


Table 4 Odds ratio (OR) using multinomial logistic regression of change in inclination towards biomedical career

\begin{tabular}{|c|c|c|c|c|}
\hline \multirow[t]{2}{*}{ Factors } & \multicolumn{2}{|c|}{$\begin{array}{l}\text { Increase inclination towards } \\
\text { research }\end{array}$} & \multicolumn{2}{|c|}{$\begin{array}{l}\text { Decrease inclination towards } \\
\text { research }\end{array}$} \\
\hline & Bivariate (OR) & $\begin{array}{l}\text { Multivariate }^{\mathrm{a}} \\
\text { (OR) }\end{array}$ & $\begin{array}{l}\text { Bivariate } \\
(\mathrm{OR})\end{array}$ & $\begin{array}{l}\text { Multivariate } \\
\text { (OR) }\end{array}$ \\
\hline $\begin{array}{l}\text { Had an experience during medical school that put off respondent from } \\
\text { research by final year }\end{array}$ & $0.52(0.25-1.11)$ & & $1.43(0.82-2.46)$ & \\
\hline $\begin{array}{l}\text { Read biomedical journals at least once every } 6 \text { months during medical } \\
\text { school by final year }\end{array}$ & $1.77(0.99-3.17)$ & & $0.66(0.40-1.08)$ & \\
\hline Presence of role model during medical school by final year & $1.71(0.92-3.16)$ & & $0.73(0.39-1.35)$ & \\
\hline Increased desire to learn more about biostatistics & $2.02(1.18-3.46)^{*}$ & $2.58(1.38-4.84)^{*}$ & $0.86(0.54-1.36)$ & $0.87(0.50-1.51)$ \\
\hline Increased knowledge of ease of manipulation of statistics & $1.69(1.01-2.83)^{*}$ & & $0.86(0.55-1.35)$ & \\
\hline $\begin{array}{l}\text { Increased confidence in analyzing the data to find association between } \\
\text { two variables }\end{array}$ & $1.18(0.75-1.86)$ & $1.10(0.66-1.83)$ & $0.47(0.31-0.70)^{*}$ & $0.49(0.31-0.78)^{*}$ \\
\hline $\begin{array}{l}\text { Increased confidence in analyzing the data to find correlation between } \\
\text { two variables }\end{array}$ & $1.57(0.99-2.48)$ & & $0.61(0.41-0.90)^{*}$ & \\
\hline Increased confidence in evaluating diagnostic tests & $1.57(0.96-2.57)$ & & $0.76(0.51-1.12)$ & \\
\hline Increased understanding of research ethics terms in journal articles. & $0.96(0.61-1.51)$ & & $0.62(0.42-0.92)^{*}$ & \\
\hline $\begin{array}{l}\text { Increased confidence in applying for an approval from an Institutional } \\
\text { Review Board/ethics committee }\end{array}$ & $1.37(0.84-2.25)$ & & $0.74(0.49-1.12)$ & \\
\hline Increased understanding of study design terms in journal articles. & $1.04(0.63-1.70)$ & & $0.60(0.39-0.92)^{*}$ & \\
\hline
\end{tabular}

adjusted for gender, age and institution

*statistically significant OR

acknowledged that this could be a potential confounding factor in our analysis, hence the conclusion of this study to be made with caution.

\section{Conclusions}

With caution of the limitations of this study, we conclude that most medical students do not change their inclination towards research after completing their undergraduate medical training. This finding also suggests that for medical students to improve their inclination towards research, the medical curricula should be changed to incorporate more research activities, and greater attention should be given to building students' knowledge of the research process and methods of statistical analysis [17]. In addition, good research role models should be made available to interested students [18], which may require an incentive system to encourage researchers to be role models. As training clinicians who can play his/her part in medical research is important for the future of medicine, medical schools could also consider selecting medical students based on their past research activities and their prior interest in research at time of application.

\section{Abbreviations}

BGU: Ben-Gurion University (BGU) Israel; JCU: James Cook University; NTNU: Norwegian University of Science and Technology (NTNU); NUS: National University of Singapore (NUS); OR: Odds Ratio; PhD: Doctorate of Philosophy; PUC: Pontificia Universidad Catolica de Chile (PUC);

SD: Standard Deviation; USA: United States of America

\section{Acknowledgements}

We would like to thank the medical students who participated in this study.

\section{Availability of data and materials}

The datasets used and/or analysed during the current study are available from the corresponding author on reasonable request.

\section{Authors' contributions}

$\mathrm{TCH}$ coordinated the overall project, collected data, supervised and revised the manuscript, SN ran the analysis of the study, CC helped planned the statistical approach to analyzing the data, SKY helped in drafting the manuscript, GCHK coordinated the research at Yong Loo Lin School of Medicine, National University of Singapore and revised the manuscript, SBT was involved in the initial conceptualization of the project, RM helped coordinate the collection of data Duke- National University of Singapore and revised the manuscript, FA coordinated the research at Pontificia Universidad Catolica de Chile, Santiago, Chile, AS coordinated the research at Norwegian University of Science and Technology (NTNU), Norway, AB coordinated the research at Ben-Gurion University, Beer-Sheva, Israel, TW coordinated the research at James Cook University Australia and revised the manuscript, TO coordinated the research at Duke University, USA and revised the manuscript. All authors read and approved the final manuscript.

\section{Ethics approval and consent to participate}

Study participation was voluntary and informed written consent obtained from each student. Individual ethics approval was also obtained where appropriate from each country's institution.

Singapore

Approval was obtained from the NUS Institutional Review Board (NUS-IRB

Ref No 08-274) for both the NUS and DUKE-NUS sites in Singapore. Australia

Approval was obtained from James Cook University Human Research Ethics Committee, approval numbers H5595 \& H3031.

USA

Approval was obtained from DUKE Medicine Institutional Review Board for Clinical Investigations, protocol number 00013362

Israel

Approval was obtained from the Ethics Committee of Soroka University Medical Center, approval number 10345. 
Norway

The Regional Committee for Medical Research Ethics permitted this study to be exempt from ethics approval and also confirmed that according to Norwegian legislation informed consent was not mandatory.

Chile

Approval was obtained from Comité de Ética de Investigación de la Escuela de Medicina, Pontificia Universidad Católica de Chile, protocol number CE\# $87 / 09$.

\section{Competing interests}

The authors declare that they have no competing interests.

\section{Publisher's Note}

Springer Nature remains neutral with regard to jurisdictional claims in published maps and institutional affiliations.

\section{Author details}

'Duke-NUS Medical School, National University of Singapore, Singapore, Singapore. ${ }^{2}$ The University of Wollongong, Wollongong, Australia. ${ }^{3}$ Saw Swee Hock School of Public Health, National University of Singapore, Singapore, Singapore. ${ }^{4}$ Pontificia Universidad Catolica de Chile, Santiago, Chile. ${ }^{5}$ Norwegian University of Science and Technology (NTNU), Trondheim, Norway. ${ }^{6}$ Ben-Gurion University, Beer-Sheva, Israel. ${ }^{7}$ James Cook University, Townsville, Australia. ${ }^{8}$ Duke University Medical Centre, Durham, NC, USA.

Received: 24 November 2017 Accepted: 20 April 2018

Published online: 02 May 2018

\section{References}

1. The European Commission's Scientific Panel for Health (SPH). Vision on 'better research for better health: horizon 2020; 2016.

2. Ley TJ, Rosenberg LE. The physician-scientist career pipeline in 2005: build it, and they will come. JAMA. 2005;294(11):1343-51.

3. Guelich JM, Singer BH, Castro MC, Rosenberg LE. A gender gap in the next generation of physician-scientists: medical student interest and participation in research. JIM. 2002;50(6):412-8.

4. Tan SB, Koh GCH, Ding YW, Malhotra R, Ha TC, Pietrobon R, Kusumaratna R, Tie RN, Cunha G, Martins H, Seim A, Altermatt F, Biderman A, Puoane T, Carvalho E, Ostbye T. Inclination towards a research career among first year medical students: an international study. South East Asian J Med Educ. 2011;5:49-59.

5. Cluver J, Book S, Brady K, Back S, Thornley N. Engaging medical students in research: reaching out to the next generation of physician-scientists. Acad Psychiatry. 2014;38(3):345-9.

6. Chapman SJ, Glasbey JC, Khatri C, Kelly M, Nepogodiev D, Bhangu A, Fitzgerald JE. Promoting research and audit at medical school: evaluating the educational impact of participation in a student-led national collaborative study. BMC Med Educ. 2015;15(1):1.

7. Rosenkranz SK, Wang S, Hu W. Motivating medical students to do research: a mixed methods study using self-determination theory. BMC Med Educ. 2015:15(1):1.

8. Houlden RL, Raja JB, Collier CP, Clark AF, Waugh JM. Medical students' perceptions of an undergraduate research elective. Med Teach. 2004;26(7): 659-61.

9. Evered DC, Anderson J, Griggs P, Wakeford R. The correlates of research success. Br Med J. 1987;295(6592):241-6.

10. Andriole DA, Whelan AJ, Jeffe DB. Characteristics and career intentions of the emerging MD/PhD workforce. JAMA. 2008:300(10):1165-73.

11. Al-Nuaimi $Y$, McGrouther G, Bayat A. Modernising medical careers and factors influencing career choices of medical students. Br J Hosp Med. 2008; 69(3):163-6.

12. Huda N, Yousuf S. Career preference of final year medical students of Ziauddin medical university. Educ Health. 2006;19(3):345-53.

13. Siemens DR, Punnen S, Wong J, Kanji N. A survey on the attitudes towards research in medical school. BMC Med Educ. 2010;10(1):4.

14. Kassebaum DG, Szenas PL, Ruffin AL, Masters DR. The research career interests of graduating medical students. Acad Med. 1995;70(9):848-52.

15. Koenig JA. Comparison of medical school performances and career plans of students with broad and with science-focused premedical preparation. Acad Med. 1992;67(3):191-6.
16. KhadarooRG ROD. Are clinician-scientists an endangered species? Barriers to clinician-scientist training. Clin Invest Med. 2002;25(6):260-1.

17. Burazeri G, Civljak M, llakovac V, Jankovic S, Majica-Kovacevic T, Nedera O, et al. Survey of attitudes and knowledge about science in medical students in Southeast Europe. BMJ. 2005;331(7510):195-6.

18. Strong MJ, Busing N, Goosney DL, Harris KA, Horsley T, Kuzyk A, et al. The rising challenge of training physician-scientists: recommendations from a Canadian National Consensus Conference. Acad Med. 2017;16(10): 0000000000001857

\section{Ready to submit your research? Choose BMC and benefit from:}

- fast, convenient online submission

- thorough peer review by experienced researchers in your field

- rapid publication on acceptance

- support for research data, including large and complex data types

- gold Open Access which fosters wider collaboration and increased citations

- maximum visibility for your research: over $100 \mathrm{M}$ website views per year

At BMC, research is always in progress.

Learn more biomedcentral.com/submissions 